\title{
Proper motion of very low mass stars and brown dwarfs in the Pleiades cluster ${ }^{\star}$
}

\author{
E. Moraux ${ }^{1}$, J. Bouvier ${ }^{1}$, and J. R. Stauffer ${ }^{2}$ \\ 1 Laboratoire d'Astrophysique, Observatoire de Grenoble, Université Joseph Fourier, BP 53, \\ 38041 Grenoble Cedex 9, France \\ http://www-laog.obs.ujf-grenoble.fr/activites/starform/formation.html \\ 2 SIRTF Science Center, Caltech, Pasadena, CA 91125, USA
}

Received 16 November 2000 / Accepted 30 November 2000

\begin{abstract}
We report proper motion measurements for 25 very-low mass (VLM) star and brown dwarf (BD) candidates of the Pleiades cluster previously identified by Bouvier et al. (1998). Proper motions are measured with an accuracy of 9 mas/yr, compared to an expected tangential motion of about 50 mas/yr for Pleiades members. Of the 25 candidates, 15 have a membership probability of $95 \%$ or more and 7 are rejected as being field dwarfs. The 3 remaining candidates exhibit independent evidence for membership (lithium absorption or long-term proper motion). From the firm identification of Pleiades VLM and BD members, the cluster's substellar mass function is revised to $\mathrm{d} N / \mathrm{d} M \propto M^{-0.5}$ in the mass range from 0.04 to $0.3 M_{\odot}$.
\end{abstract}

Key words. stars: low-mass, brown dwarfs - stars: luminosity function, mass function - Galaxy: open clusters and associations: individual: Pleiades

\section{Introduction}

The determination of the stellar mass function is an important challenge for various domains of astrophysics such as, e.g., the star formation process, the structure and evolution of the Galaxy, the dynamical evolution of stellar clusters and stellar systems, etc. This function describes the relative number of stars per unit mass and is usually approximated by power-law segments of the form $\mathrm{d} N / \mathrm{d} M$ $\propto M^{-\alpha}$ in various mass domains. While its shape is relatively well constrained from solar-type to massive stars ( $\alpha \simeq 2.3-2.7$ for $M \geq 1 M_{\odot}$, Salpeter 1955, Scalo 1998 and references therein), it is more uncertain for low-mass stars $\left(\alpha \simeq 1.0-2.0\right.$ for $0.3 \leq M \leq 1 M_{\odot}$, Kroupa 2000 and references therein) and, up to a couple of years ago, was still unexplored at very low masses in both the stellar $\left(0.08 \leq M \leq 0.3 M_{\odot}\right)$ and substellar $\left(M \leq 0.08 M_{\odot}\right)$ regimes. The determination of the substellar mass function is indeed one of the main motivations for the recent and exploding quest for free-floating brown dwarfs (see, e.g., Oppenheimer et al. 2000 for a review).

An estimate of the mass function (MF) in the upper part of the substellar domain $\left(\sim 0.04-0.08 M_{\odot}\right)$ now exist for the Pleiades cluster (Bouvier et al. 1998:

Send offprint requests to: E. Moraux, e-mail: moraux@laog.obs.ujf-grenoble.fr

* Based on observations obtained at Canada-France-Hawaii Telescope. $\alpha \simeq 0.6 \pm 0.15$; Martín et al. 1998, 2000: $\alpha \simeq 0.5-1.0$, Hambly et al. 1999: $\alpha \sim 0.7$ ) and has recently been derived for field brown dwarfs in the solar neighbourhood (Reid et al. 1999: $\alpha \simeq 1.0-2.0$ depending on the local stellar birthrate). Determinations of the substellar mass function have also been reported for several star forming regions (e.g. Comeron et al. 2000; Luhman \& Rieke 1999; Hillenbrand \& Carpenter 2000) with, however, the additional difficulty of varying extinction within the molecular cloud which makes more problematic the derivation of an unbiased sample of very low-mass young objects that can be used to build the substellar MF.

Estimates of the substellar mass function of the Pleiades cluster rely on deep, wide-field photometric surveys that identify substellar candidates from their location in optical color-magnitude diagrams (CMD). One of the major concern regarding these samples of very low-mass (VLM) and brown dwarf (BD) candidates is the degree of contamination by foreground late-type field dwarfs, which may lie in the same region of the CMD as the Pleiades low-mass members. Bouvier et al. (1998) thus estimated at about $30 \%$ the level of contamination of their candidate sample by field dwarfs. Some of the BD candidates have actually been confirmed through the "lithium test" (Rebolo et al. 1992) but, even with the largest telescopes, only the brightest substellar candidates are amenable to this test (Stauffer et al. 1998). Other Pleiades BD candidates originally identified from their 
location in an $(I, R-I)$ CMD have later been rejected on the basis of their discrepant near-IR colors (Martín et al. 2000). However, it is currently unclear whether all contaminating field stars can be unambiguously recognized in a near-IR CMD.

A powerful way to recognize true Pleiades members among the photometric candidates is to measure their proper motion. Proper motion studies should allow us to pick out members with a high degree of confidence because the cluster's peculiar motion is large compared to the non-member field stars $\left(\mu_{\alpha} \cos \delta=19.15 \pm 0.23 \mathrm{mas} / \mathrm{yr}\right.$, $\mu_{\delta}=-45.72 \pm 0.18$, Robichon et al. 1999) and the intrinsic velocity dispersion of the cluster is small ( $\sim 1 \mathrm{mas} / \mathrm{yr}$, Van Leeuwen 1980). Such a large motion can be measured from sets of sharp images separated by only a few years and as soon as a candidate is recognized as a Pleiades member on the basis of its kinematics, its status (VLM star or BD) directly follows from its photometric properties.

We therefore obtained in September 1999 new images for 25 of the 26 VLM star and BD candidates of the Pleiades originally observed by Bouvier et al. (1998) in December 1996. In Sect. 2, we describe how proper motion was derived for each candidate from the 2 sets of images separated by nearly 3 years. In most cases, the results presented in Sect. 3 allow us to unambiguously identified Pleiades members and they are compared with other diagnostics of Pleiades membership. In Sect. 4, we discuss whether previous estimates of the Pleiades substellar function has to be revised in the light of these new results and briefly discuss the kinematics of very low-mass members of the cluster with respect to their formation mechanism.

\section{Observations and astrometric reduction}

The first set of images, from which VLM and BD candidates were identified, was obtained by Bouvier et al. (1998) in December 1996 with the CFHT 8K wide-field camera. Of the 26 identified candidates, 25 were reobserved in September 1999 with the CFHT 12K camera. Both instruments have the same pixel size $\left(0.21^{\prime \prime}\right)$ and the images were obtained under comparable seeing conditions $\left(0.9^{\prime \prime}\right.$ and $0.7^{\prime \prime}$, respectively). In 1999, the exposure time was adjusted so as to provide similar signal-to-noise ratio on the candidates as on the 1996 images. Astrometry was performed on $I$-band images from the 2 epochs.

The principle of the astrometric procedure we used to measure the proper motion of the candidates is as follows. We first measure the position $\left(x_{96}, y_{96}\right)$, $\left(x_{99}, y_{99}\right)$ of the candidate on the two sets of images using IRAF/CENTER. On the same images, we also measure the positions of typically 10 point-like objects, presumably background field stars, located within an angular radius of about $3^{\prime}$ from the candidate on the CCD. These stars are then used as relative astrometric references to compute the spatial transformation function that maps 1999 coordinates to the 1996 reference frame using the IRAF/IMMATCH package. The rms uncertainty associated to the spatial transformation, $\sigma_{\mathrm{t}}$, is computed by the IRAF task as well as the residuals for each astrometric reference star. In case the residuals for a reference star is significantly larger than $\sigma_{\mathrm{t}}$, the object is discarded and the transformation recomputed.

This transformation is applied to $\left(x_{99}, y_{99}\right)$ in order to project the 1999 coordinates of the candidate in the 1996 reference frame: $\left(x_{99}, y_{99}\right) \longrightarrow\left(x_{99 \rightarrow 96}, y_{99 \rightarrow 96}\right)$. The displacement of the object in pixels between 1996 and 1999 is simply given by:

$\Delta x=x_{99 \rightarrow 96}-x_{96}=(\Delta t) \mu_{x}$

$\Delta y=y_{99 \rightarrow 96}-y_{96}=(\Delta t) \mu_{y}$

where $\Delta t$ is the time lag between the two epochs and $\mu_{x}$, $\mu_{y}$ are the relative proper motions along the $x$ and $y$ axes of the CCD in the 1996 reference frame. The precision of the measurement is $\sigma_{\Delta x}^{2}=\sigma_{\Delta y}^{2}=2 \sigma_{x, y}^{2}+\sigma_{\mathrm{t}}^{2}$, where $\sigma_{x, y}$ is the rms error on the measurement of the position of the candidate on the CCD and $\sigma_{\mathrm{t}}$ the error associated with the spatial transformation from one epoch to the other. We evaluated $\sigma_{x, y} \simeq 0.07$ pixels by measuring the positions of the same objects on consecutive $I$-band images of the same field. Tests were run with the IRAF/GEOMAP package in order to minimize $\sigma_{\mathrm{t}}$. We found that a third degree polynomial transformation yields the best results with $\sigma_{\mathrm{t}} \simeq 0.05$ pixels. We verified a posteriori that the proper motion measured for each candidate was not unduely sensitive either to the degree of the polynomial transformation or to the number of astrometric reference stars used to compute the transformation.

The displacement $\left(\mu_{x}, \mu_{y}\right)$ in pixels/yr has still to be converted into a displacement on the sky $\left(\mu_{\alpha} \cos \delta, \mu_{\delta}\right)$ in mas/yr by calibrating the spatial scale and orientation of the 1996 coordinates system. The calibration was done using PRIAM (Procédure de Réduction d'Images AstroMétriques), a software package developed by A. Fienga and J. Berthier at the IMCCE (Institut de Mécanique Céleste et de Calcul des Éphémérides). This software calculates the transformation which converts pixel positions $(x, y)$ into J2000 celestial coordinates $(\alpha, \delta)$ for a set of astrometric standards. For each candidate field, we measured on the image the position of typically 10 stars belonging to the USNO2 catalogue whose astrometric accuracy is of order 500 mas. PRIAM then computes the coefficients $\mathrm{A} 0, . ., \mathrm{B} 2$ of the transformation:

$\alpha=A 0+A 1 x+A 2 y$

$\delta=B 0+B 1 x+B 2 y$

from which we obtain:

$\mu_{\alpha} \cos \delta=A 1 \mu_{x}+A 2 \mu_{y}$

$\mu_{\delta}=B 1 \mu_{x}+B 2 \mu_{y}$ 
The rms error on these coefficients is $\sim 0.1 \mathrm{mas} /$ pixel, and can be neglected compared to $\sigma_{x, y}$ and $\sigma_{\mathrm{t}}$. Hence, the final uncertainty on the proper motion measurement is

$\sigma_{\mu_{\alpha} \cos \delta}^{2} \sim \sigma_{\mu_{\delta}}^{2} \sim \frac{p i x^{2}}{\Delta t^{2}}\left(2 \sigma_{x, y}^{2}+\sigma_{\mathrm{t}}^{2}\right)$

which typically amounts to 9 mas/yr rms for a pixel scale $p i x=205$ mas and $\Delta t=2.8 \mathrm{yr}$.

\section{Results}

The proper motions of the 25 Pleiades VLM star and BD candidates are listed in Table 1 and illustrated in a vector point diagram (VPD) in Fig. 1. The field stars used as astrometric references scatter around the origin of the diagram since they have been selected on the basis of a negligible proper motion. In contrast, most of the Pleiades candidates fall within $3 \sigma$ of the expected cluster's mean motion. Following the method outlined by Sanders (1971) we compute a membership probability for each candidate as follows:

$p=\frac{f_{\mathrm{c}}\left(\mu_{\alpha} \cos \delta, \mu_{\delta}\right)}{f_{\mathrm{f}}\left(\mu_{\alpha} \cos \delta, \mu_{\delta}\right)+f_{\mathrm{c}}\left(\mu_{\alpha} \cos \delta, \mu_{\delta}\right)}$

where $f_{\mathrm{c}}\left(\mu_{\alpha} \cos \delta, \mu_{\delta}\right)$ and $f_{\mathrm{f}}\left(\mu_{\alpha} \cos \delta, \mu_{\delta}\right)$ are the vector point distributions of the members and field stars respectively. We assumed $f_{\mathrm{c}}$ is a bivariate normal function and we found

$$
\begin{aligned}
f_{\mathrm{c}}\left(\mu_{\alpha} \cos \delta, \mu_{\delta}\right)=0.04 \exp & \left\{-\frac{1}{2}\left[\left(\frac{\mu_{\alpha} \cos \delta-21.5}{7.7}\right)^{2}\right.\right. \\
+ & \left.\left.\left(\frac{\mu_{\delta}+40.1}{8.5}\right)^{2}\right]\right\}
\end{aligned}
$$

by fitting a 2-D Gaussian, plus an assumed uniform distribution for field stars, on the vector-points distribution of the VLM and BD candidates. The field stars distribution is then $f_{\mathrm{f}}=3.310^{-4}$. We also tried to reproduce the distribution of both Pleiades candidates and astrometric references in the VPD by adding a 2-D Gaussian centered on $(0,0)$ to the fitting function. This leads to a dispersion of $\sim 4.5$ mas/yr for the astrometric references. Membership probabilities computed for the candidates by either method are very similar because the distributions of candidates and astrometric references do not overlap in the VPD.

The membership probability of the candidates is listed in Table 2. We also list in this table other diagnostics of Pleiades membership obtained by Stauffer et al. (1998) and Martín et al. (2000), namely: $E W(\mathrm{Li}), E W\left(\mathrm{H}_{\alpha}\right)$, radial velocity, and $(I-K)$ index. We consider the 15 candidates for which we found $p \geq 95 \%$ as Pleiades members. It is interesting to note that two of these confirmed members (CFHT-PL-7 a very low-mass Pleiades star, and CFHT-PL-25, the least massive brown dwarf) do not exhibit $\mathrm{H} \alpha$ in emission according to Martin et al. (2000). In addition to these 15 highly probable Pleiades members, CFHT-PL-12 has a membership probability of $88 \%$ and lithium has been detected in its spectrum (Stauffer et al. 1998) which makes it a bona fide Pleiades brown dwarf.

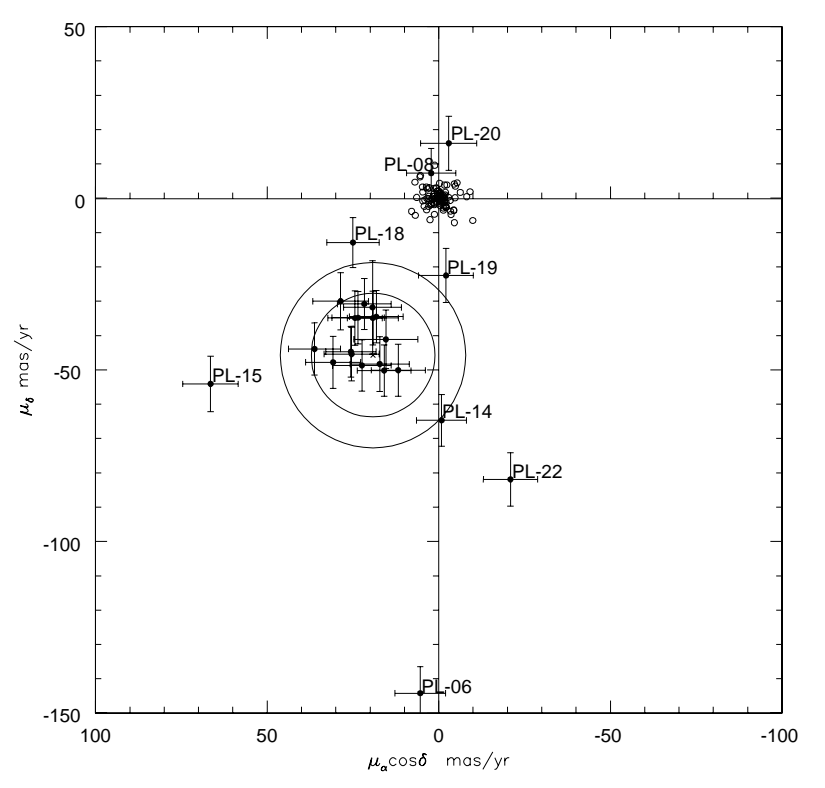

Fig. 1. Proper motion vector point diagram for objects located in Pleiades fields. Filled dots show VLM and BD candidates and open circles the field stars used as astrometric references. North is up and East is left. The cluster proper motion is indicated by a cross and two circles centered on this value are drawn. The radius is equal to $2 \sigma$ and $3 \sigma$ for the small and the big circle respectively where $\sigma$ corresponds to the 2D-Gaussian dispersion of the candidates in this diagram (see text)

We could not measure the proper motion of CFHT-PL-3 (= HHJ 22) because it is located both at the edge of a CCD and at the edge of the camera field of view which makes the computation of the spatial transformation between the two epochs unreliable. However, the long-term proper motion of HHJ 22 has been measured by Hambly et al. (1999) with an epoch difference of nearly 40 years and indicates highly probable Pleiades membership. We therefore consider this candidate as a cluster member.

The remaining 8 candidates (CFHT-PL 6, 8, 14, 15, $18,19,20,22)$ have very low membership probabilites $(p \leq 20 \%)$. Of these, CFHT-PL-14 and 18 had already been rejected as a non-members based on the absence of lithium absorption in their spectrum and CFHT-PL-19, 20 , and 22 were suspected non-members based on their peculiar location in near-IR CMDs. The low membership probability we measure for these objects confirmed that they are non-members. The 3 remaining candidates (CFHT-PL 6, 8, and 15) deserve further discussion.

CFHT-PL-6's proper motion is high and more than $10 \sigma$ away from the cluster's mean motion. On the one hand, CFHT-PL-6 does not exhibit $\mathrm{H} \alpha$ emission (Martín et al. 2000) but this alone does not rule out membership since its twin, CFHT-PL-7, also lacks $\mathrm{H} \alpha$ emission but is a confirmed Pleiades member from its proper motion. On the other hand, CFHT-PL-6 lies significantly above the Pleiades ZAMS which led Bouvier et al. (1998) to suggest that it could be a nearly equal mass binary which, in turn might affect its short term proper motion. If it is indeed an equal mass binary, then the components of 
Table 1. Proper motion of Pleiades VLM and BD candidates. The Pleiades cluster proper motion is $\overline{\mu_{\alpha} \cos \delta}=19.15 \pm$ $0.23 \mathrm{mas} / \mathrm{yr}$ and $\overline{\mu_{\delta}}=-45.72 \pm 0.18 \mathrm{mas} / \mathrm{yr}$

\begin{tabular}{lccccc}
\hline Name & Other id. & $\begin{array}{c}\mu_{\alpha} \cos \delta \pm \sigma_{\mu_{\alpha} \cos \delta} \\
(\operatorname{mas} / \mathrm{yr})\end{array}$ & $\begin{array}{c}\mu_{\delta} \pm \sigma_{\mu_{\delta}} \\
(\operatorname{mas} / \mathrm{yr})\end{array}$ & $\begin{array}{c}\left(\mu_{\alpha} \cos \delta-\overline{\mu_{\alpha} \cos \delta}\right) \\
\sigma_{\mu_{\alpha} \cos \delta}\end{array}$ & $\frac{\left(\mu_{\delta}-\overline{\mu_{\delta}}\right)}{\sigma_{\mu_{\delta}}}$ \\
\hline CFHT-PL-1 & & $22.4 \pm 8.5$ & $-48.7 \pm 7.5$ & 0.4 & 0.4 \\
CFHT-PL-2 & & $15.4 \pm 9.3$ & $-41.1 \pm 8.5$ & 0.4 & 0.5 \\
CFHT-PL-3 & HHJ22 & - & - & & \\
CFHT-PL-4 & & $17.2 \pm 8.6$ & $-48.3 \pm 8.0$ & 0.2 & 0.3 \\
CFHT-PL-5 & & $18.2 \pm 7.8$ & $-34.5 \pm 7.6$ & 0.1 & 1.5 \\
CFHT-PL-6 & & $5.4 \pm 7.4$ & $-144.2 \pm 7.7$ & 1.9 & 12.8 \\
CFHT-PL-7 & & $19.2 \pm 7.4$ & $-34.9 \pm 7.8$ & 0.01 & 1.4 \\
CFHT-PL-8 & & $2.21 \pm 7.2$ & $7.31 \pm 7.2$ & 2.35 & 1.4 \\
CFHT-PL-9 & & $19.3 \pm 8.4$ & $-31.8 \pm 13.6$ & 0.02 & 1.9 \\
CFHT-PL-10 & & $28.6 \pm 8.1$ & $-30.0 \pm 8.3$ & 1.2 & 0.04 \\
CFHT-PL-11 & Roque 16 & $25.4 \pm 8.0$ & $-45.4 \pm 7.8$ & 0.8 & 0.2 \\
CFHT-PL-12 & & $36.2 \pm 7.6$ & $-43.9 \pm 7.6$ & 2.2 & 0.6 \\
CFHT-PL-13 & Teide 2 & $11.8 \pm 7.9$ & $-50.1 \pm 7.6$ & 0.9 & 2.5 \\
CFHT-PL-14 & & $-0.8 \pm 7.3$ & $-64.7 \pm 7.5$ & 2.7 & 1.0 \\
CFHT-PL-15 & & $66.5 \pm 8.1$ & $-54.1 \pm 8.1$ & 5.8 & 2.0 \\
CFHT-PL-16 & & $21.7 \pm 7.8$ & $-30.8 \pm 7.4$ & 0.3 & 0.3 \\
CFHT-PL-17 & & $30.8 \pm 8.0$ & $-47.8 \pm 7.6$ & 1.5 & 4.5 \\
CFHT-PL-18 & & $25.0 \pm 7.6$ & $-12.9 \pm 7.3$ & 0.8 & 2.9 \\
CFHT-PL-19 & & $-2.1 \pm 8.0$ & $-22.5 \pm 7.9$ & 2.6 & 7.8 \\
CFHT-PL-20 & & $-2.9 \pm 8.2$ & $16.0 \pm 7.9$ & 2.7 & 1.4 \\
CFHT-PL-21 & Calar 3 & $23.5 \pm 7.6$ & $-34.8 \pm 7.6$ & 0.6 & 4.6 \\
CFHT-PL-22 & & $-20.9 \pm 7.9$ & $-81.9 \pm 7.8$ & 5.1 & 0.6 \\
CFHT-PL-23 & & $15.9 \pm 7.8$ & $-50.2 \pm 7.5$ & 0.4 & 0.1 \\
CFHT-PL-24 & Roque 7 & $24.4 \pm 7.9$ & $-34.9 \pm 7.9$ & 0.7 & 0.9 \\
CFHT-PL-25 & & $25.6 \pm 7.3$ & $-44.7 \pm 7.4$ & & \\
\hline
\end{tabular}

${ }^{\dagger}:$ CFHT-PL-3 is located close to the edge of a CCD and at the edge of the mosaic's field of view. This leads to large distorsions that prevent us from deriving a reliable measurement of its proper motion. ${ }^{\ddagger}$ : The measurement error on $\mu_{\delta}$ is larger than average for CFHT-PL-9 due to charge transfer problems on one of the CCDs of the mosaic that smears the stellar profile.

CFHT-PL-6 would be substellar and therefore amenable to the lithium test. We also find that CFHT-PL-6 was about $0.1 \mathrm{mag}$ brighter in the $I$-band in 1996 than in 1999. This level of photometric variablity is typical of late-M dwarfs (Martín et al. 1998) but might also occur in substellar objects (Bailer-Jones \& Mundt 1999). Based primarily on its highly discrepant proper motion and pending additional observations of this object, we tentatively conclude that CFHT-PL-6 is most likely a foreground M dwarf and not a cluster member.

CFHT-PL-8 has a vanishingly small membership probability with a proper motion close to that of background field stars. It had previously been considered as a probable member based on $E W(\mathrm{H} \alpha)=14.6 \AA$, a spectral type of dM5.6 and IR colors consistent with membership (Martín et al. 2000). These properties, however, are not inconsistent with it being a low-mass field dwarf. Even the extremely short rotational period of 0.401 days recently derived by Terndrup et al. (1999) is not unexpected for very low-mass dwarfs (Delfosse et al. 1997). We thus conclude that this candidate is not a cluster member.

CFHT-PL-15 is puzzling in several ways. There is little doubt that this late-type object (Sp.T. M7) is a Pleiades brown dwarf since lithium has been detected in its spectrum (Stauffer et al. 1998). Yet, the tangential motion we measure is clearly different from that of Pleiades members. Moreover, Stauffer et al. (1998) find that it exhibits unusual colors that locate it slightly below the Pleiades ZAMS. From the analysis of HST images, Martín et al. (2000) found evidence for high residuals after PSF subtraction, which might indicate the presence of an unresolved companion at a separation less than $0.22^{\prime \prime}$ and about 3 mag fainter than CFHT-PL-15. It is thus conceivable that the short-term peculiar tangential motion we measure results from orbital motion in a binary system or photometric variability of one or both components. The latter appears more likely since, with an estimated substellar mass, the maximum displacement of the photocenter due to orbital motion would be less than 25 mas over 3 years while we measure $\sim 135$ mas. However, CFHTPL-15 does not seem to be a photometric binary from its location in a color-magnitude diagram. For the time being, we thus consider CFHT-PL-15 as a highly probable Pleiades brown dwarf based on the presence of lithium in its spectrum. 
Table 2. Candidates membership criteria. The $E W(\mathrm{Li})$ are from Stauffer et al. (1998) and the others tests have been performed by Martín et al. (2000). In Col. 8, we give the membership probability of the candidates based on their proper motion. Our final assessment regarding membership is indicated Col. 9

\begin{tabular}{|c|c|c|c|c|c|c|c|c|}
\hline Name & other id. & $\begin{array}{c}E W(\mathrm{Li}) \\
(\AA)\end{array}$ & $\mathrm{H}_{\alpha}$ & $V_{\text {rad }}$ & $I-K$ & Martin et al. & $\begin{array}{l}\text { Iembership } \\
\text { prob. (\%) }\end{array}$ & Conclusion \\
\hline CFHT-PL-1 & & & yes & & yes & yes & 98.6 & yes \\
\hline CFHT-PL-2 & & & yes & & yes & yes & 98.9 & yes \\
\hline CFHT-PL-3 & HHJ22 & & & & yes & yes & - & yes \\
\hline CFHT-PL-4 & & & & & yes & yes? & 98.6 & yes \\
\hline CFHT-PL-5 & & & yes & & yes & yes & 98.8 & yes \\
\hline CFHT-PL-6 & & & no & & yes & yes? & $\ll 1$ & no? \\
\hline CFHT-PL-7 & & & no & & yes & yes? & 98.9 & yes \\
\hline CFHT-PL-8 & & & yes & & yes & yes & $\ll 1$ & no \\
\hline CFHT-PL-9 & & $<0.05$ & yes & yes & yes & yes & 98.4 & yes \\
\hline CFHT-PL-10 & & $<0.05$ & yes & yes & yes & yes & 95.7 & yes \\
\hline CFHT-PL-11 & Roque 16 & 0.5 & yes & yes & yes & yes & 98.7 & yes \\
\hline CFHT-PL-12 & & 0.8 & yes & yes & yes & yes & 87.7 & yes \\
\hline CFHT-PL-13 & Teide 2 & 0.6 & yes & yes & yes & yes & 96.7 & yes \\
\hline CFHT-PL-14 & & $<0.1$ & no & no & & no & 1.3 & no \\
\hline CFHT-PL-15 & & 0.5 & yes & yes & yes & yes & $\ll 1$ & yes \\
\hline CFHT-PL-16 & & 1.2 & yes & yes & yes & yes & 98.1 & yes \\
\hline CFHT-PL-17 & & & yes & & yes & yes & 96.1 & yes \\
\hline CFHT-PL-18 & & no & yes & & yes & no & 17.6 & no \\
\hline CFHT-PL-19 & & & & & no & no? & 4.0 & no \\
\hline CFHT-PL-20 & & & no & & no & no & $\ll 1$ & no \\
\hline CFHT-PL-21 & Calar 3 & yes & yes & yes & yes & yes & 98.7 & yes \\
\hline CFHT-PL-22 & & & & & no & no & $\ll 1$ & no \\
\hline CFHT-PL-23 & & & & & yes & yes & 98.1 & yes \\
\hline CFHT-PL-24 & Roque 7 & & & & & yes & 98.7 & yes \\
\hline CFHT-PL-25 & & & no & & yes & yes & 98.7 & yes \\
\hline
\end{tabular}

\section{Discussion}

The firm identification of Pleiades VLM stars and brown dwarfs from their kinematics provides a clean, albeit small, sample of substellar objects from which a more reliable estimate of the substellar mass function of the cluster can be derived. In addition, these are the first proper motion measurements obtained with an accuracy of better than 10 mas/yr for very low mass Pleiades members and this allows us to start to investigate the intrinsic velocity distribution of brown dwarfs in the cluster. These two aspects are briefly discussed below after the status of the objects contaminating the photometric sample is investigated.

\subsection{The nature of the contaminating objects}

Seven objects of the original photometric sample are found to be probable non members. These objects are located in the same region of the $(R-I, I) \mathrm{CMD}$ as bona fide Pleiades VLM stars and brown dwarfs. Their proper motions in Fig. 1 do not seem to be uniformly distributed but tend to scatter over the lower left quadrant of the vector-point diagram. In order to investigate the status of the contaminating objects we used the kinematical model of the Galaxy developed by Robin \& Crézé (1986). From the model, we constructed a synthetic sample of field stars assumed to be observed in the galactic direction of the Pleiades cluster and covering a magnitude range
$I=16-20$ and a color range $R-I=1.7-2.7$. The sample thus computed contains nearly $10^{4}$ stars of which 13 lie in the same location of the CMD as the Pleiades VLM and $\mathrm{BD}$ candidates. According to the model, these 13 objects are late M-dwarfs (M 6-M 9) distributed over a distance between 70 and $170 \mathrm{pc}$ and their proper motions indeed scatter preferentially over the lower left quadrant of the VPD with an amplitude up to 120 mas/yr. The same eccentric distribution of proper motions is seen for the whole sample of $10^{4}$ stars and results from the effect of galactic differential rotation in the direction of the Pleiades cluster. We thus conclude that the photometric candidates rejected as being non Pleiades member based on their proper motion are a mixture of foreground and background late-M dwarfs of the galactic disk.

\subsection{The Pleiades substellar IMF}

From accurate proper motion measurements, we have identified 7 out of 25 VLM and BD candidate members as probable field stars. In addition, the lowest mass candidate of Bouvier et al.'s (1998) sample, CFHT-PL-26, was not included in this study but Martín et al. (2000) classified it as a non-member based on its spectral properties (discrepant pseudo-continuum indices and lack of $\mathrm{H}_{\alpha}$ emission). The overall level of contamination of the photometric sample by field dwarfs is thus $8 / 26=31 \%$, 
close to the original estimate of Bouvier et al. (1998) based on statistical arguments.

Do these results modify the earlier estimate of the substellar mass function of the Pleiades cluster? Of the 18 photometric candidates with a mass between 0.04 and $0.08 M_{\odot}$ in the original sample (CFHT-PL-9 to 26), 6 are rejected here as non-members. Thus counting 12 confirmed objects in this mass range, a power law fit to the mass function between $0.3 M_{\odot}$ and $0.04 M_{\odot}$ yields: $\mathrm{d} N / \mathrm{d} M \propto M^{-0.51 \pm 0.15}$, i.e., slightly shallower than the earlier estimate $\left(\mathrm{d} N / \mathrm{d} M \propto M^{-0.60 \pm 0.15}\right)$. This slight revision is not very significant considering that the errors are still dominated by Poisson noise from small samples.

These results do not account for binarity. CFHT-PL6 (whose membership is however in doubt, see above), 12 , and 16 appear to lie on the binary sequence of the cluster in a color-magnitude diagram. If these 3 objects are nearly equal mass cluster binaries, one finds $\mathrm{d} N / \mathrm{d} M \propto$ $M^{-0.65 \pm 0.15}$.

\subsection{Kinematics of Pleiades VLM stars and BDs}

The $\mu_{\alpha} \cos \delta$ and $\mu_{\delta}$ distributions of the Pleiades VLM and $\mathrm{BD}$ candidates are illustrated in Fig. 2. A Gaussian fit to the distributions of the confirmed Pleiades members leads to dispersions of $\sigma_{\mu_{\alpha} \cos \delta}=7.2 \mathrm{mas} / \mathrm{yr}$ and $\sigma_{\mu_{\delta}}=8.5 \mathrm{mas} / \mathrm{yr}$ that are similar to the expected measurement error. Hence, we find no evidence for an intrinsic dispersion in the distribution of tangential velocities of the VLM and BD Pleiades members. This is consistent with the hypothesis that these VLM and substellar objects formed in the same way as stellar cluster members did, i.e., from the collapse of isolated, very low mass molecular cloud cores. Indeed, the internal dispersion of Pleiades stars is $\sim 1$ mas/yr (Van Leeuwen 1980).
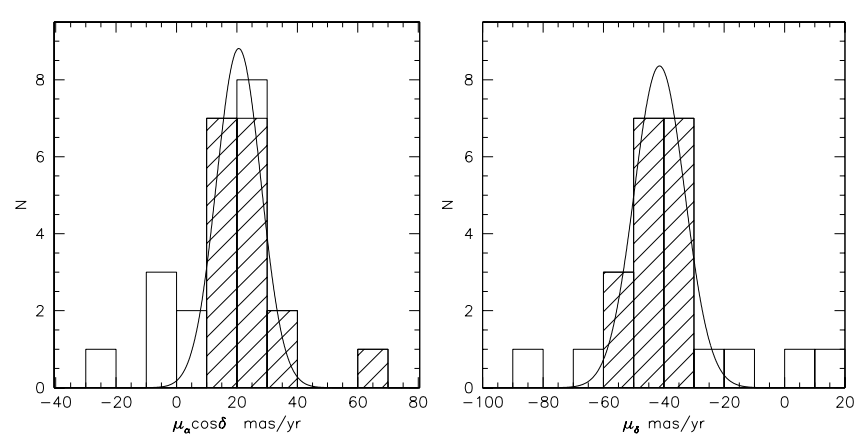

Fig. 2. Proper motion distributions for program stars. The hatched histogram refers to candidates confirmed as being Pleiades members and the solid curve is a Gaussian fit to their proper motion distribution

However, owing to the difficulty of triggering the collapse of molecular cores whose mass is much lower than the typical Jeans mass $\left(\simeq 0.7 M_{\odot}\right.$, Clarke et al. 2000), alternative models have been proposed for the formation of brown dwarfs. Burkert et al. (1997) have shown that collapsing molecular cores are prone to multiple fragmentation that eventually leads to the formation of small- $N$ protoclusters, including a number of very low mass fragments. Following dynamical interactions within the protocluster, the least massive fragments are preferentially ejected with typical velocities of order of a few $\mathrm{km} \mathrm{s}^{-1}$ (Burkert, priv. comm.) and may thus become isolated brown dwarfs (Klessen \& Burkert 2000). Alternatively, Lin et al. (1998) proposed that isolated brown dwarfs may form as a result of an encounter between protostars with massive disks. The encounter leads to the formation of an unbound tidal tail which contains part of the initial disk's mass and may later condensed to form an isolated substellar object. Here again, the typical ejection velocities are of order of a few $\mathrm{km} \mathrm{s}^{-1}$.

At a distance of 118 pc, a tangential velocity of $1 \mathrm{~km} \mathrm{~s}^{-1}$ corresponds to a tangential motion of about 2 mas/yr. Proto brown bwarfs ejected with this velocity in the early stages of cluster formation, about 120 Myr ago, would have now drifted by several tens of degrees away from the cluster. If these BD formation models are correct, the sample we have observed close to the cluster's center must represent a tiny fraction of the primordial BDs, namely those which populated the low tail of the distribution of ejection velocities. In such a case, the slope of the substellar mass function of the Pleiades cluster might currently be largely underestimated.

\section{Conclusion}

A sample of very low-mass stars and brown dwarfs have been firmly identified in the Pleiades cluster on the basis of their proper motion. With 12 confirmed objects with a mass less than $0.08 M_{\odot}$ distributed over an area of 2.5 square degrees close to the cluster's center, the Pleiades mass function is estimated to be $\mathrm{d} N / \mathrm{d} M \propto M^{0.51 \pm 0.15}$ in the mass domain ranging from 0.04 to $0.3 M_{\odot}$. Taking individual components of suspected substellar binaries into account leads to $\mathrm{d} N / \mathrm{d} M \propto M^{0.65 \pm 0.15}$. The main source of uncertainty on these estimates now lies in the unknown radial distribution of brown dwarfs relative to stars in the cluster. As a group, the identified brown dwarfs exhibit no intrinsic velocity dispersion. This suggests that they have formed from the collapse of isolated low mass molecular cores. However, we cannot rule out that this sample of brown dwarfs represent only a tiny fraction of the brown dwarf population originally formed in the cluster and which might have escaped since then either due to dynamical ejection at the time of their formation or during the subsequent dynamical evolution of the cluster. Additional studies covering a much larger fraction of the cluster's area are needed to settle this issue.

Acknowledgements. We thank A. Fienga for allowing us access to PRIAM before public release, A. Robin for help in using the Besancon's galactic model, and C. Clarke for discussions on cluster kinematics. 


\section{References}

Bailer-Jones, C. A. L., \& Mundt, R. 1999, A\&A, 348, 800

Bouvier, J., Stauffer, J. R., Martin, E. L., et al. 1998, A\&A, 336,490

Burkert, A., Bate, M., Matthew, R., \& Bodenheimer, P. 1997, MNRAS, 289, 497

Klessen, R., \& Burkert, A. 2000, ApJS, 128, 287

Clarke, C. J., Bonnell, I. A., \& Hillenbrand, L. A. 2000, Protostars and Planets IV, ed. V. Manning, A. P. Boss, \& S. S. Russell, 151

Comeron, F., Neuhäuser, R., \& Kaas, A. A. 2000, A\&A, 359, 269

Delfosse, X., Forveille, T., Perrier, C., \& Mayor, M. 1998, A\&A, 331,581

Hambly, N. C., Hawkins, M. R. S., \& Jameson, R. F. 1993, A\&AS, 100, 607

Hambly, N. C., Hodgkin, S. T., Cossburn, M. R., \& Jameson, R. F. 1999, MNRAS, 303, 835

Hillenbrand, L. A., \& Carpenter, J. M. 2000, ApJ, 540, 236

Kroupa, P. 2000, MNRAS, in press

Lin, D. N. C., Laughlin, G., Bodenheimer, P., \& Rózyczka, M. 1998, Science, 281, 2025

Luhman, K. L., \& Rieke, G. H. 1999, ApJ, 525, 440
Martin, E. L., Zapatero Osorio, M. R., \& Rebolo, R. 1996, ASP Conf. Ser., 109, 615

Martin, E. L., Basri, G., Gallegos, J. E., et al. 1998, ApJ, 499, L61

Martin, E. L., Brandner, W., Bouvier, J., et al. 2000, ApJ, in press

Oppenheimer, B. R., Kulkarni, S. R., Stauffer, J. R. 2000, Protostars and Planets IV, ed. V. Manning, A. P. Boss, \& S. S. Russell, 1313

Rebolo, R., Martin, E. L., \& Magazzù, A. 1992, ApJ, 389, L83

Reid, I. N., Kirkpatrick, J. D., Liebert, J., et al. 1999, ApJ, 521,613

Robin, A., \& Crézé, M. 1986, A\&A, 157, 71

Robichon, N., Arenou, F., Mermilliod, J. C., \& Turon, C. 1999, A\&A, 345, 471

Sanders, W. L. 1971, A\&A, 14, 226

Salpeter, E. E. 1955, ApJ, 121, 161

Scalo, J. 1998, in The Stellar Initial Mass Function, ed. G. Gilmore, D. Howell, ASP Conf. Ser., 142, 201

Stauffer, J. R., Schultz, G., \& Kirckpatrick, J. D. 1998, ApJ, 499, L199

Terndrup, D. M., Krishnamurthi, A., Pinsonneault, M. H., \& Stauffer, J. R. 1999, ApJ, 118, 1814

van Leeuwen, F. 1980, Stars Clusters, IAU Symp., 85, 157 